\title{
Confined Water Determines Transport Properties of Guest Molecules in Narrow Pores
}

\author{
Anh Phan ${ }^{1}$, David R. Cole ${ }^{2}$, R. Gregor Weiß $\beta^{3,4}$, Joachim Dzubiella ${ }^{3,4}$ and Alberto Striolo ${ }^{1, *}$ \\ ${ }^{1}$ Department of Chemical Engineering, University College London, London, WC1E 7JE, UK \\ ${ }^{2}$ School of Earth Sciences, The Ohio State University, Columbus, Ohio 43210, USA \\ ${ }^{3}$ Institut für Physik, Humboldt-Universität zu Berlin, Newtonstrasse 15, D-12489 Berlin, Germany
}

${ }^{4}$ Soft Matter and Functional Materials, Helmholtz-Zentrum Berlin, Hahn-Meitner Platz 1, D-14109 Berlin, Germany

\begin{abstract}
We computed the transport of methane through 1-nm wide slit-shaped pores carved out of solid substrates. Models for silica, magnesium oxide, and alumina were used as solid substrates. The pores were filled with water. The results show that the methane permeability through the hydrated pores is strongly dependent on the solid substrate. Detailed analysis of the simulated systems reveals that local properties of confined water, including its structure, and, more importantly, evolution of solvation free energy and hydrogen bond structure are responsible for the pronounced differences observed. The simulations are extended to multi-component systems representative of natural gas, containing methane, ethane, and $\mathrm{H}_{2} \mathrm{~S}$. The results show that all pores considered have high affinity for $\mathrm{H}_{2} \mathrm{~S}$, moderate affinity for methane, and low affinity for ethane. The $\mathrm{H}_{2} \mathrm{~S} /$ methane transport selectivity through hydrated alumina pores is comparable, or superior, to that reported for existing commercial membranes. A multi-scale approach was then implemented to demonstrate that a Smoluchowski one-dimensional model is able to reproduce the molecular-level results for short pores when appropriate values for the local self-diffusion coefficients are used as input parameters. We propose that the model can be extended to predict methane transport through uniform hydrated pores of macroscopic length. When verified by experiments, our simulation results could have important implications in applications such as natural gas sweetening and predictions of methane migration through hydraulically fractured shale formations.

* Author to whom correspondence should be addressed: $\underline{\text { a.striolo@ucl.ac.uk }}$

Keywords: solvation free energy, confined fluids, molecular simulation, aqueous systems, permeability
\end{abstract}




\section{Introduction}

The separation of natural gas into its components is required for a variety of technological applications. For example, natural gas, as extracted, contains methane, ethane, a few higher molecular weight hydrocarbons, all of which find use in the petro-chemical industry, but also water, carbon dioxide, hydrogen sulfide $\left(\mathrm{H}_{2} \mathrm{~S}\right)$ and other compounds that are deleterious for the aforementioned uses. Sweetening of the gas (removal of $\mathrm{H}_{2} \mathrm{O}, \mathrm{H}_{2} \mathrm{~S}$ and $\mathrm{CO}_{2}$ ) is needed to avoid pipe corrosion during transportation and to enhance the heating power of the gas, while the recovery of ethane and higher molecular weight hydrocarbons is desirable for upgrade processes such as cracking and polymerization. Industrially, natural gas sweetening is often performed via expensive and energy-intensive distillation processes, ${ }^{1,2}$ although membranes are being designed for improving upon such processes. ${ }^{3}$ Research continues to be performed to better engineer the membranes and improve their selectivity and capacity. ${ }^{4}$ State-of-the-art membranes include those obtained using rubbery polyamide-polyether block copolymers, commercially available as Pebax, which allow the simultaneous separation of $\mathrm{CO}_{2}$ and $\mathrm{H}_{2} \mathrm{~S}$ from $\mathrm{CH}_{4}$; these membranes are known to swell in the presence of liquid water, with negative impact on the membrane selectivity. ${ }^{5}$ Rubbery materials containing $\mathrm{H}_{2} \mathrm{~S}$-philic groups are sensitive to penetrant $\mathrm{H}_{2} \mathrm{~S}$ at high feed pressures, which causes swelling and plasticization. ${ }^{6}$ As a consequence, rubbery composite membranes typically do not endure high-pressure operations, a problem for natural gas sweetening, in which the feed gas pressure is often higher than $4 \mathrm{MPa}^{5}$ Membranes that are not polymeric (e.g., those obtained from graphene sheets), could allow to achieve excellent performance, but this requires well-controlled manufacturing of the porous materials. The resultant materials can be very sensitive to the presence of moisture in the gaseous mixture and may lose mechanical integrity at high pressure.

From a different point of view, the presence of methane in drinking water has been documented in several parts of the world, most notably in the Marcellus region in the North East of the United States. ${ }^{7}$ Because of the widespread implementation of hydraulic fracturing for the stimulation of shale formations in the region during the last 10-20 years, a strong debate is ongoing regarding the source of the methane detected in drinking water. Possible sources include microbial and thermogenic ones. Two facts stand out: 
shale formations are fractured at depths of at least $1,500 \mathrm{~m}$, and the permeability of shale rocks to fluids and gases is extremely low (of the order of a few millidarcies or much less). These observations are commonly identified as supporting evidence for the low probability of drinking water contamination due to hydraulic fracturing. The low recovery of hydraulic fracturing water and its potential impact on hydrocarbon production are key issues that remain poorly constrained. For example, recovery of frack fluid from the Haynesville shale is very low, roughly 5\% whereas for the Barnett and Marcellus recovery can reach as high as $50 \% .{ }^{8}$ Imbibition of fracturing fluid into the nanoporous rock matrix has been identified as a possible mechanism for fluid loss and reservoir damage. ${ }^{9,10}$ To substantiate or refute this claim, it is critical to better understand the transport of low molecular weight hydrocarbons, especially methane, through narrow pores representative of those found in sub-surface shale formations. It is of particular interest to understand whether methane can transport across narrow pores filled with water, especially because recent computational results have suggested that the methane solubility in confined water can be one order of magnitude larger than that measured in bulk water. Other reports are also supporting enhanced gas solubility due to confinement effects. ${ }^{11-13}$

The computational research presented here reveals a somewhat surprising outcome that small hydrocarbons (e.g., methane) can diffuse through narrow pores filled with water. This can have important implications in understanding the migration of carbon-bearing fluids in the sub-surface. Moreover, the prediction of hydration structure within narrow pores seems to lead to significant chemical selectivity, which could be exploited for the separation of gases, e.g., in natural gas sweetening. For the latter application, it is notable that the solid materials used for the present study are obtained from rather stable structures representative of minerals found in the sub-surface, and therefore are expected to be chemically stable for time scales shorter than those at which mineral dissolution processes become relevant. 


\section{Materials and Methods}

We conducted extensive equilibrium and non-equilibrium molecular dynamics (MD) simulations. The models and algorithms implemented have been described at length in our prior contributions, ${ }^{14,15}$ and therefore only a short summary is provided herein.

Solid supports. We considered slit-shaped pores obtained from three model materials: silica, alumina, and $\mathrm{MgO}$, shown in Figure 1. Details about three model materials are described elsewhere. ${ }^{11,16-21}$ All the nonbridging oxygen atoms on silica and alumina surfaces were protonated; ${ }^{17,18}$ the $\mathrm{MgO}$ surfaces were not hydroxylated. ${ }^{22}$ The latter is an over-simplification of reality, but it allows us to understand, computationally, how the fluid structure under confinement determines the membrane performance. The $\mathrm{X}$ and $\mathrm{Y}$ dimensions of the three surfaces were of $51.7 \times 100.8,46.9 \times 90.7$, and $52.6 \times 105.1 \dot{\mathrm{A}}^{2}$, for silica, alumina, and $\mathrm{MgO}$, respectively.

Force fields. The CLAYFF force field was implemented to simulate the solid substrates. ${ }^{23}$ The silicon, aluminum and oxygen atoms were held at fixed positions while the surface hydroxyl hydrogen atoms were allowed to vibrate. All $\mathrm{MgO}$ atoms were kept rigid. The transferable potentials for phase equilibria (TraPPE) force field was implemented to model methane and ethane. ${ }^{24}$ The rigid SPC/E model was used to simulate water. ${ }^{25}$ The potential parameters developed by Kamath et al. were used to model $\mathrm{H}_{2} \mathrm{~S} .{ }^{26}$ Bond lengths and angles of water were kept fixed by implementing the SETTLE algorithm. ${ }^{27}$ Non-bonded interactions were modeled by means of dispersive and electrostatic forces. The electrostatic interactions were modeled by the Coulombic potential, with long-range corrections treated using the particle mesh Ewald (PME) method. ${ }^{28}$ Dispersive interactions were modeled by 12-6 Lennard-Jones (LJ) potentials. The LJ parameters for unlike interactions were determined by Lorentz-Berthelot combining rules ${ }^{29}$ from the values of like components. The cutoff distance for all interactions was set to $9 \AA$.

Simulation set up. The simulation box is periodic in the three directions. The Y dimension of the simulation boxes reflects the periodicity of the solid crystalline substrates; the $\mathrm{X}$ and $\mathrm{Z}$ dimensions were set to $172.7-42.9,211.2-33.8$, and $201.4 \dot{\mathrm{A}}-33.1 \dot{\mathrm{A}}$ for silica, alumina, and pores, respectively. The pore width in all the three systems was set at $10 \dot{\mathrm{A}}$ (the pore width is defined as the shortest center-to-center 
distance between surface oxygen atoms across the pore volume). Due to periodic boundary conditions, the nanopores are effectively infinite along the $\mathrm{Y}$ direction. Conversely, the pores are finite along the $\mathrm{X}$ direction, along which they are exposed to the feed (e.g., methane) and permeate (water + vacuum) phases.

The simulation setup mimics the one implemented by Balbuena et al. ${ }^{30}$ and by Mizukami et al., ${ }^{31}$ adapted to investigate the effect of hydration water on selectivity. The initial configurations were built with water molecules filling the slit pore. The number of water molecules was fixed at 4500 for three systems. These molecules were sufficient to fill the pore volume and form thin layers on the solid substrate outside of the pores. As the simulation progresses and methane flows across the pore, the water film remains only on the low-pressure side of the solid membrane (see Figure 1). The hydration water within the three pores does not show marked differences as the simulations progress. Outside of the pore, one wall was artificially constructed using helium atoms, to separate a feed phase on the left side of the pore from the permeate phase on the right side of the pore. This wall was necessary because of the periodicity of the simulation box along the $\mathrm{X}$ direction. The helium atoms interact only weakly with other molecules in the system, guaranteeing that the wall does not affect the results concerning the transport of fluids across the pores. ${ }^{32}$ Methane was then inserted, randomly, on the left side of the pore, the feed region. The number of methane molecules inserted was of 3000, 3700, and 4000 for the silica, $\mathrm{MgO}$, and alumina systems, respectively. Different amounts of methane were necessary to drive methane through these membranes at a similar flux. In other words, instead of imposing equal pressure drops for the three systems simulated, we opted for changing the pressure drop so as to achieve an almost equal methane flux for the three systems simulated. The feed region is built of equal volume for silica, $\mathrm{MgO}$ and alumina systems. The pressures in the feed regions were calculated using the Peng-Robinson equation of states using the molecular density of pure methane as input. ${ }^{33}$ As the simulations progress, methane molecules transport through the water-filled pore from the feed to the permeate phase. To evaluate the membrane performance by the approach at a constant pressure gradient ${ }^{34}$ we carried out the following actions: every 30 ns remove every molecule that has entered the permeate phase and add an equal number of molecules to the feed 
phase, thus we ensure that the pressure gradient is kept constant during the simulations. The number of molecules in the membrane and the number of molecules that passes to the permeate phase are monitored against time to obtain the flux across the membrane.

Algorithms. The MD simulations were conducted using the package GROMACS. ${ }^{35,36}$ Simulations were performed in the canonical ensemble (NVT), where the number of particles $(\mathrm{N})$, the simulation volume $(\mathrm{V})$, and the temperature $(\mathrm{T})$ are kept constant. The simulated temperature was maintained at $300 \mathrm{~K}$ by a Nose-Hoover thermostat ${ }^{37,38}$ with a relaxation time of 100 fs. The equations of motion were solved by implementing the leapfrog algorithm ${ }^{39}$ with 1.0 fs time steps. The total simulation time was $600 \mathrm{~ns}$ for each system.

Data analysis. The results have been analyzed taking advantage of numerous recent computational advancements to calculate membrane permeability, diffusion, free energy profiles, solvation free energy, and mean first passage time (MFPT) profiles.

Membrane permeability. We calculate the mole flux of gas transport $(J)$ across three different pores as: $J=\frac{\Delta Q_{t} / \Delta t}{A}$

where $\Delta Q_{t} / \Delta t$ is the slope of the fitted straight line for cumulative number of methane molecules in the permeate region $\left(Q_{t}\right)$ as a function of time $t$ and $A$ is the cross-sectional area available for gas penetration perpendicular to the direction of diffusion. ${ }^{40}$ We can approximate the observed methane permeability $(K)$ by the simple formula ${ }^{41}$

$K=\frac{J \times l}{p_{1}-p_{2}}$

where $p_{1}$ is the applied pressure in the feed phase, $p_{2}$ is zero according to the protocol we implemented at constant pressure gradient, and $l$ is the length of pore in the $\mathrm{X}$ direction.

Transport diffusion. We calculate the transport diffusion from the time lag defined as the intercept of the extrapolated linear part of $Q_{t}$ on the time axis. This type of time lag analysis was first proposed by Daynes $^{41}$ and then refined by Barrer $^{42}$, and is widely used experimentally to obtain transport 
diffusivities. ${ }^{43}$ The approach cannot distinguish between the transport mechanisms that occur in the pore. The transport diffusion coefficient of methane is related to the time lag via a simple expression ${ }^{41}$

$D_{t}=\frac{l^{2}}{6 \theta}$

where $\theta$ is the time lag.

$\underline{\text { Self-diffusion. }}$ Three simulations were carried out for computing the planar self-diffusivity of methane inside three $2 \mathrm{D}$ periodically infinite hydrated pores under equilibrium MD simulations for $25 \mathrm{~ns}$. The systems were built with water molecules filling the slit pore. Ten methane molecules were inserted randomly inside the pore. The mean square displacement (MSD) approach was used for self-diffusion calculations and the self-diffusion coefficient $D_{s}$ is calculated with the Einstein relation, ${ }^{44,45}$

$D_{s}=\frac{1}{2 d} \lim _{t \rightarrow \infty} \frac{\left\langle\left|r_{i}(t)-r_{i}(0)\right|^{2}\right\rangle}{t}$,

where $r_{i}(t)$ is the position of molecule $i$ at the time $t, d$ is the dimensionality of the system, and $\langle\ldots\rangle$ denotes ensemble average.

Free energy profiles. To construct the potential of mean force (PMF) calculated for one methane molecule moving across the hydrated pores, we implemented the umbrella sampling algorithm. ${ }^{46,47}$ The methane molecule was forced to remain at a given position through the hydrated pore in the $\mathrm{X}$ direction using harmonic springs of elastic constant $3000 \mathrm{~kJ} / \mathrm{mol} . \mathrm{nm}^{2}$. For each pore, 80 independent simulations were conducted, imposing that the equilibrium position changes by $0.1 \mathrm{~nm}$ from one simulation to another. At any given position the simulation was conducted for up to $8 \mathrm{~ns}$, during which time the histogram representing the position of the molecule with respect to the pore entrance was populated. ${ }^{48}$ The WHAM algorithm was then used to reconstruct the potential of mean force from combining the histograms obtained at various locations. ${ }^{47}$

Solvation free energy. Initially, we quantify water density fluctuations by calculating the probability of observing $N$ molecules within a small spherical observation volume $v$ of radius $r=3.3 \dot{\mathrm{A}}$ located at the center of the hydrated pores. The probabilities are calculated as: ${ }^{49}$ 
$P_{v}(N)=\left\langle\delta\left(N_{v}-N\right)\right\rangle=\lim _{t \rightarrow \infty} \frac{1}{t} \int_{0}^{t} d t^{\prime} \delta\left(N_{v}\left(t^{\prime}\right)-N\right)$

This distribution is related to the solvation free energy for an ideal hydrophobe through the relation ${ }^{50}$

$\Delta \mu_{v}=-\frac{1}{\beta} \ln P_{v}(0)$

where $\Delta \mu_{v}$ is the solvation free energy and $\beta$ is one over temperature times Boltzmann's constant.

MFPT. Assuming that the dynamics of methane are strictly Markovian, according to the theory of stochastic processes, the mean first passage time (MFPT) for first reaching a target distance $x_{t}$ when starting off from $x$, is given by ${ }^{51,52}$

$\tau_{f p}\left(x, x_{t}\right)=\int_{x_{t}}^{x} d x^{\prime} \frac{e^{\beta F\left(x^{\prime}\right)}}{D(x)} \int_{x^{\prime}}^{x_{r}} d x^{\prime \prime} e^{-\beta F\left(x^{\prime \prime}\right)}$,

where $x_{r}$ denotes the position of a reflective boundary.

Statistics for MFPT, which describe the time scale for the methane moving from the center of the pores to the outside of the pores, are obtained based on a series of 400 independent MD simulation runs for the system containing water filling the pores and one methane molecule at the center of the simulation box constrained in the $\mathrm{Y}-\mathrm{Z}$ plane ( $8000 \mathrm{~ns}$ of total simulation time). Results are shown below for the hydrated silica pores. Each of the 400 runs is started with random initial atomic velocities. After 500 ps of equilibration, the methane molecule at the center is allowed to move freely only along the $\mathrm{X}$ direction until it reaches either $x_{t}=-14.98 \dot{\mathrm{A}}$ or $x_{t}=63.02 \dot{\mathrm{A}}$.

By differentiating Eq. (7) we are able to obtain the local self-diffusion profile $D(x)$ from the PMF profiles and MD-derived MFPT : $: 51,52$

$D(x)=\frac{e^{\beta F(x)}}{\partial \tau_{f p}\left(x_{t}, x\right) / \partial x} \int_{x}^{x_{r}} d x^{\prime} e^{-\beta F\left(x^{\prime}\right)}$ 


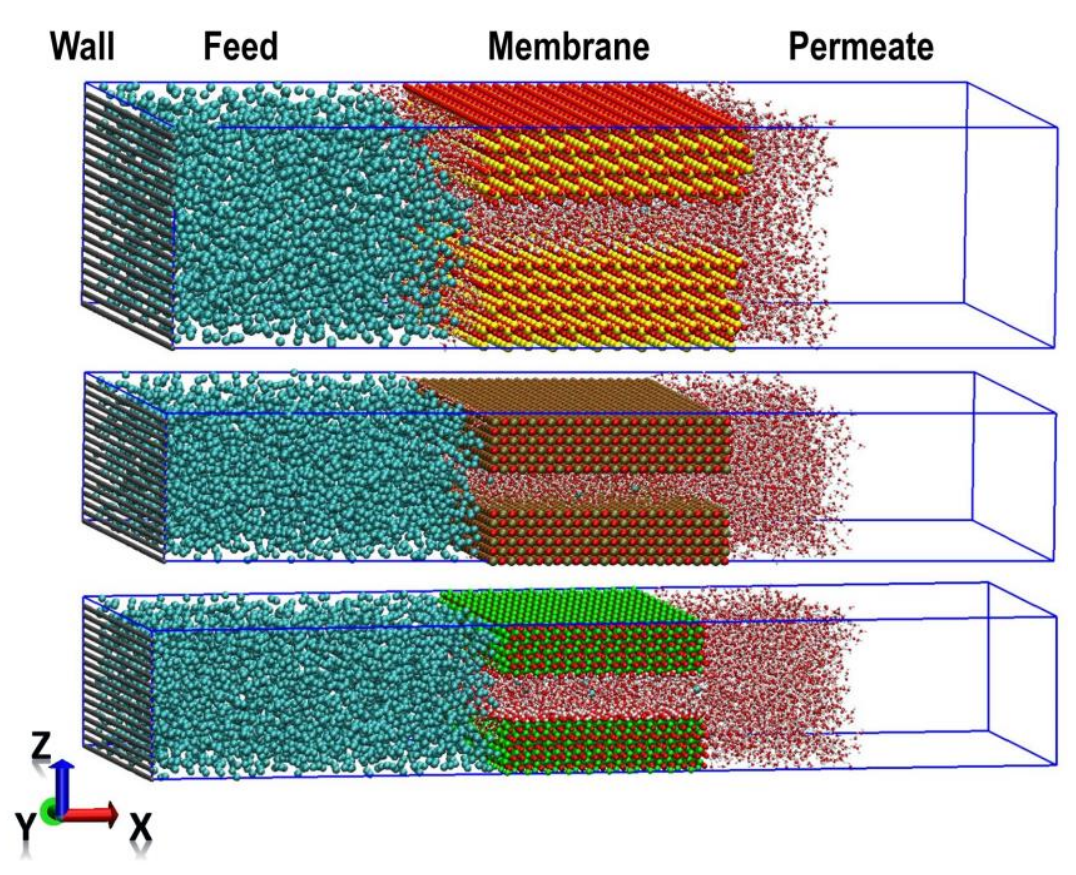

Figure 1. Representative simulation snapshots of the initial configurations for methane transport through the silica (top panel), $\mathrm{MgO}$ (middle panel) and alumina (bottom panel) narrow pores saturated with water. Gray, red, white, cyan, yellow, tan, and green spheres represent helium, oxygen, hydrogen, methane, silicon, magnesium, and aluminum atoms, respectively. The pressure drop across the membranes is maintained constant following the procedure described in Materials and Methods.

\section{Results}

\section{Transport behavior}

In Figure 1 we represent the three substrates considered for this study. In Table 1 we report the transport properties obtained for methane through the three hydrated pores. The results show that the methane flux across the three hydrated pores is similar, although the pressure drop is rather different in the three cases. The resultant permeability changes by a factor of 2 in the three substrates. For comparison, we calculated the permeability of methane through the same pores considered in Table 1, but when water is not present. The results, reported in Supporting Information, show that the permeability of methane through the pores is much larger, by about 4 orders of magnitude, when the pores are not full of water. Those results also show that the permeability is the highest through the $\mathrm{Al}_{2} \mathrm{O}_{3}$ pores and the smallest through the $\mathrm{SiO}_{2}$ pores, which is the opposite trend compared to the results obtained for the hydrated pores. This suggests that the 
presence of hydration water dramatically changes the transport properties of molecules present as guests in the water-filled pores.

Table 1. Transport properties for methane molecules through narrow pores saturated with water.

\begin{tabular}{ccccc}
\hline \hline Material & $\begin{array}{c}\text { Pressure Drop } \\
\boldsymbol{\Delta P}(\mathbf{M P a})\end{array}$ & $\begin{array}{c}\text { Flux } \\
\boldsymbol{J}\left(\mathbf{m o l} / \mathbf{m}^{\mathbf{2}} \mathbf{s}\right)\end{array}$ & $\begin{array}{c}\text { Membrane } \\
\text { Permeability } \\
\boldsymbol{K} \times \mathbf{1 0}^{\mathbf{9}} \\
\left(\mathbf{m o l m} / \mathbf{m}^{\mathbf{2}} \mathbf{s M P a}\right)\end{array}$ & $\begin{array}{c}\text { Transport Diffusion } \\
\mathbf{C o e f f i c i e n t} \\
\boldsymbol{D}_{\boldsymbol{t}} \times \mathbf{1 0}^{\mathbf{1 0}}\left(\mathbf{m}^{\mathbf{2}} \mathbf{s}\right)\end{array}$ \\
\hline Silica & $72.2 \pm 0.2$ & $72.4 \pm 1.5$ & $5.2 \pm 0.1$ & $7.8 \pm 0.5$ \\
\hline $\begin{array}{c}\text { Magnesium } \\
\text { Oxide }\end{array}$ & $120.2 \pm 0.3$ & $69.6 \pm 1.6$ & $3.1 \pm 0.1$ & $5.9 \pm 0.2$ \\
\hline Alumina & $173.1 \pm 0.3$ & $72.9 \pm 1.5$ & $2.0 \pm 0.1$ & $4.0 \pm 0.7$ \\
\hline \hline
\end{tabular}

To quantify the effects just summarized, we calculated the free energy profile for one methane molecule as it is forced to traverse the three hydrated pores while maintaining its lateral coordinates constant in the center of the hydrated pores. The corresponding potential of mean force (PMF) profiles, as can be seen in Figure 2A, exhibit pronounced oscillations, with minima of the free energy that correspond to positions in which fewer water molecules are found on average, and maxima corresponding to locations of high molecular water density. The minima obtained in the case of the hydrated silica pores are characterized by lower free energy than in the bulk, confirming that methane is favorably attracted inside hydrated silica pores. The same was not observed for methane in hydrated $\mathrm{MgO}$ or alumina pores. These results are consistent with the prediction that methane is preferentially adsorbed in the silica pore filled by water, as we reported previously. ${ }^{15}$ 

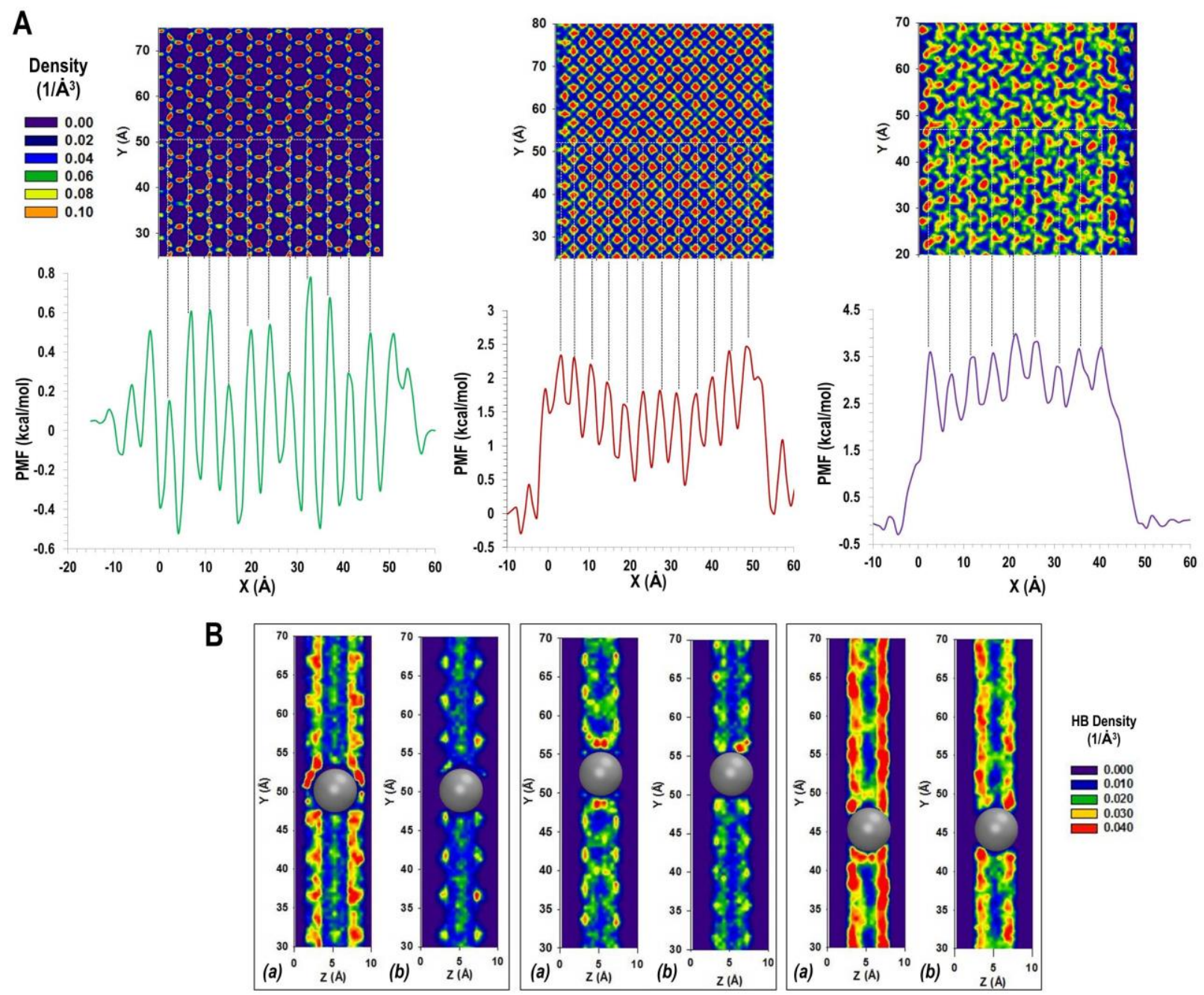

Figure 2. (A) Potential of mean force along the $\mathrm{X}$ direction as experienced by one methane molecule moving across the hydrated pores (bottom) and surface density distributions of water oxygen atoms within the first hydration layer within each of the three

pores. (B) Density distributions of water-water hydorgen bonds (HBs) found in two slabs (y-z plane) at the locations corresponding to the positions of one representative barrier $(a)$ and well $(b)$ in the potential of mean force profiles shown in (A). The gray sphere represents the constrained methane molecule. Results were obtained for silica (left panel), $\mathrm{MgO}$ (middle panel) and alumina (right panel) pores. Atomic and hydrogen bond densities are expressed in $1 / \dot{\mathrm{A}}^{3}$.

The data in Figure 2A are complementary to additional characterization data for hydration water in the three pores considered here, in particular the in-plane density distributions of water oxygen atoms found in layers parallel to the $\mathrm{X}-\mathrm{Z}$ plane at several locations along the $\mathrm{Y}$ direction within the three pores. We reported these data elsewhere, ${ }^{15}$ and we also quantified density fluctuations for water confined in the three 
pores. ${ }^{15}$ Those results demonstrated that the formation of molecular cavities within the hydration water is more likely to occur within the hydrated silica pore compared to the other two systems. We proposed the possibility that methane can adsorb in such cavities. In Figure 2B we show that when one methane molecule is adsorbed in a position corresponding to a free energy maximum $(a)$ it tends to distort the local structure of water-water hydrogen bonds (we used the geometrical criterion proposed by Marti to identify one hydrogen bond).$^{53}$ When methane is found in a free energy minimum $(b)$ lower than the bulk free energy (attractive adsorption site) the disruption of water-water hydrogen bonds appears to be minimal.

\section{$\underline{\text { Self-diffusion of methane }}$}

The results just discussed help understand why methane is preferentially adsorbed within the hydrated silica pores, but they do not clarify why the transport properties reported in Table 1 are strongly dependent on the solid substrate. To further understand the simulated systems we calculated the selfdiffusion coefficients for methane molecules at infinite dilution within the three hydrated pores. In these simulations the pores were modeled as infinite along the $\mathrm{X}$ and $\mathrm{Y}$ directions (hence the external driving force to molecular transport was absent). The results are shown in Table 2. The self-diffusion coefficients obtained for methane in the confined systems are 0.5 or less compared to those obtained for methane in bulk water at similar conditions $\left(\sim 19 \times 10^{-10} \mathrm{~m}^{2} / \mathrm{s}\right) .{ }^{54}$ This suggests that the well-ordered structure of confined water hinders methane diffusion, by a mechanism that will be discussed in detail below. We found a higher self-diffusion coefficient in the hydrated silica pore than in the other two systems, reflecting the differences in the free energy results discussed above. However, while the height of free energy barriers encountered by methane in the hydrated $\mathrm{MgO}$ and alumina pores are similar $(\sim 1.06$ and $\sim 1.05 \mathrm{kcal} / \mathrm{mol}$, respectively), the self-diffusion coefficient for methane in the hydrated $\mathrm{MgO}$ pores is larger. This suggests the possibility that methane molecules do not necessarily diffuse along a straight trajectory through the hydrated pores, possibly because water density fluctuations promote irregular trajectories for methane to diffuse through the hydrated pores. 
Table 2. Self-diffusion coefficient of one methane molecule within the three pores, within periodic boundary conditions in the two planar directions, saturated with water.

\begin{tabular}{lc}
\hline Material & $\begin{array}{c}\text { Self-Diffusion } \\
\text { Coefficient } \\
\boldsymbol{D}_{\mathbf{s}} \times \mathbf{1 0}^{\mathbf{1 0}}\left(\mathbf{m}^{\mathbf{2}} / \mathbf{s}\right)\end{array}$ \\
\hline Silica & $7.82 \pm 0.13$ \\
\hline Magnesium Oxide & $5.51 \pm 0.08$ \\
\hline Alumina & $3.26 \pm 0.05$ \\
\hline
\end{tabular}

\section{$\underline{\text { Solvation behavior }}$}

The analysis suggests that methane molecules encounter a dynamically evolving environment, rather than a static one, when adsorbed within the hydrated pores. To quantify this we followed Limmer et al. ${ }^{49}$, who proposed an algorithm to quantify the solvation free energy in water as a time-dependent property. Figure 3 displays the spatially resolved free energy distribution as experienced by a hard sphere of radius $3.3 \dot{A}$. The free energy change, $\Delta \mu_{v}$, is shown as a function of position within a $40 \times 40 \dot{\mathrm{A}}^{2}$ section within the $\mathrm{X}-$ Y plane of the simulation box. The calculations are conducted at the center of the hydrated pores and the results are averaged over $10 \mathrm{~ns}$ of simulation from an initial surface configuration $x_{\mathrm{o}}$. The results confirm that the environment provides a dynamically-changing heterogeneous solvation structure. To quantify the time scale of the dynamic fluctuations in solvation we compute the auto-correlation function: $C(t) / C(0)$, with $C(t)=\left\langle\left[\Delta \mu_{v}\left(\{x, y\}, t ; x_{0}\right)-\left\langle\Delta \mu_{v}\right\rangle\right]^{2}\right\rangle$, where the first term is the solvation free energy at $(x, y)$ averaged over the observation time $t$ from $x_{0}$, and the second term is the average solvation free energy. The results (shown in Supporting Information) suggest that a uniform solvation free energy distribution would be achieved should the averages be extended to $\sim 10 \mathrm{~ns}$ for all three pores. These results are qualitatively consistent with the observations reported by Limmer et $a .^{49}$ for liquid water in contact with metal surfaces. It is important to point out that the local solvation free energy distribution (Figure 3) does not reflect the atomic structure of the top layer of the solid surfaces (reported in details in Ref. [14]), nor the molecular structure of first or second hydration layers (middle and right panels in Figure 3). It instead 
reflects the coupling between hydrogen-bonding defect structures of the first hydration layer and the subsequent hydration layers, as suggested by Limmer et al. ${ }^{49}$
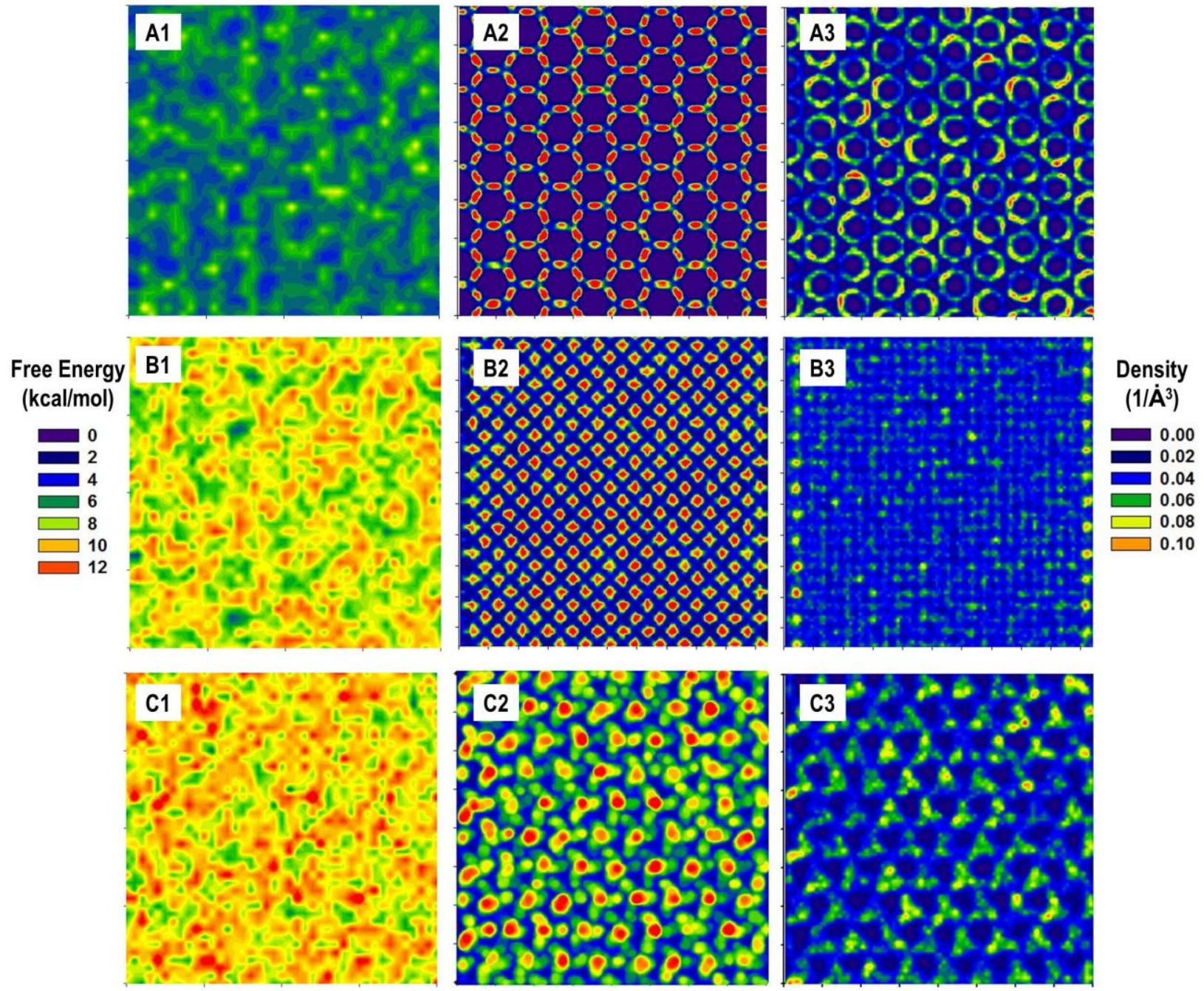

Figure 3. In-plane distribution of free energy of solvation from within a the $40 \times 40 \dot{\mathrm{A}}^{2}$ section along the $\mathrm{X}-\mathrm{Y}$ plane at the center of the hydrated pores (left). In-plane density distributions for water oxygen in the first (middle) and second (right) hydration layers formed on the pore surfaces. Results are obtained for silica (A), $\mathrm{MgO}(\mathrm{B})$ and alumia (C) pores. Solvation free energies are expressed in $\mathrm{kcal} / \mathrm{mol}$ and densities are expressed in $1 / \dot{\mathrm{A}}^{3}$. 

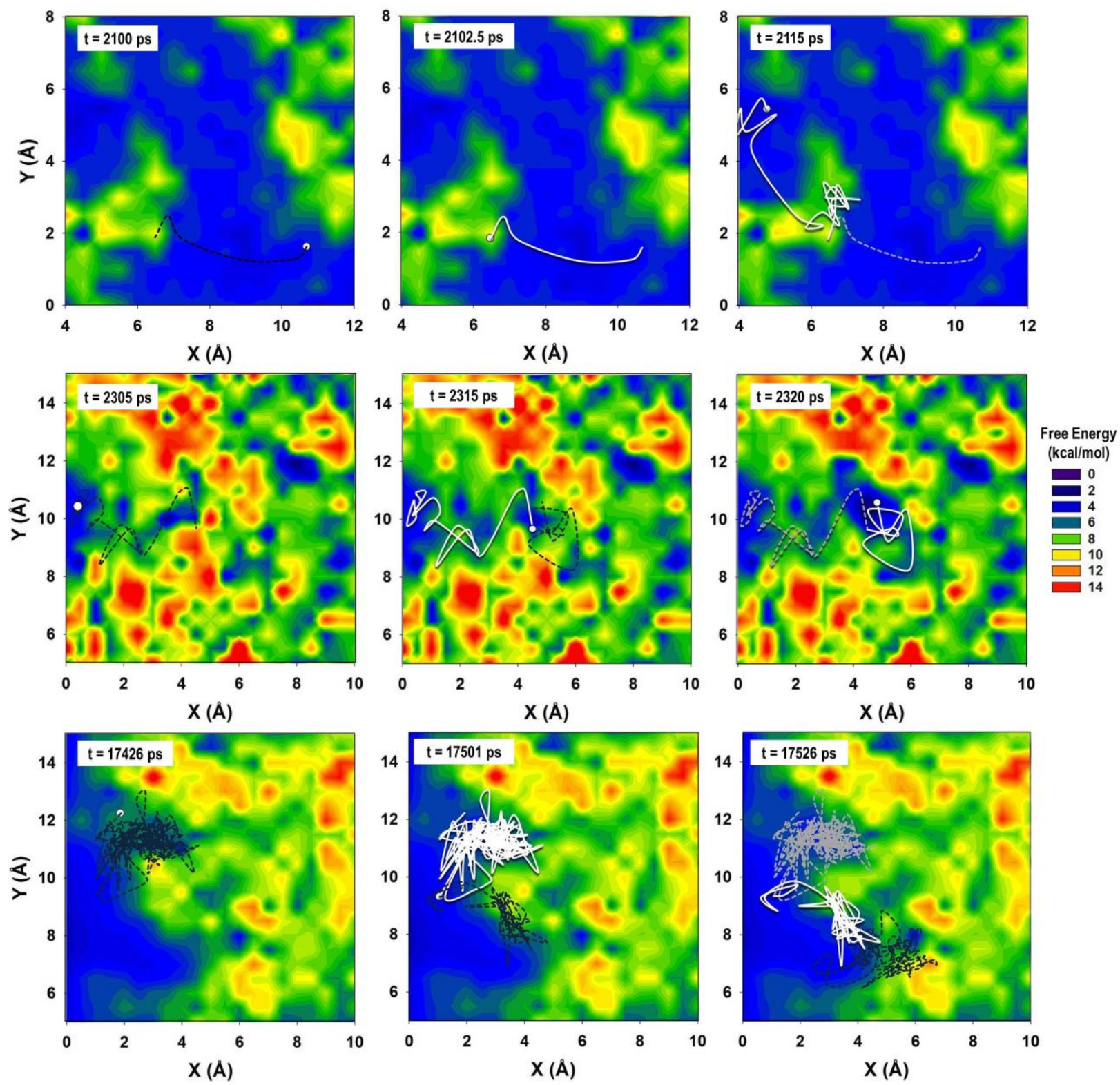

Figure 4. In-plane solvation free energy distributions overlapped with trajectories of one representative methane molecule.

Results are obtained for hydrated silica (top panels), $\mathrm{MgO}$ (middle) and alumina (bottom) pores. Regions of favorable and unfavorable solvation were determined by averaging over $2100,2102.5$, and 2115 ps for silica, 2305,2315 , and 2320 ps for $\mathrm{MgO}$, and 17426, 17501, and 17526 ps for alumina systems. Black dashed, white solid, and grey dashed lines represent the trajectories of methane for the next observation time, from the previous to the current and for the past observation time.

The heterogeneous distribution of the free energy of solvation shown in Figure 3 reinforces the possibility that averaged information such as the free energy profiles of Figure $\mathbf{2 A}$ is not sufficient to 
describe the molecular phenomena involved with the transport of methane across the hydrated pores. In fact, it is possible that local maxima and minima in the free energy landscape fluctuate with the local water density fluctuations. Thus, it is possible that the preferential path followed by methane molecules as they transport across the hydrated pores change dynamically over time. To assess this possibility, Figure 4 shows the trajectories of representative methane molecules within the hydrated pores super-imposed on the evolution of solvation free energy (SFE) distributions obtained at different observation times. It is worth pointing out that the SFE distributions change as the simulation progresses $(1 \mathrm{~ns}<\mathrm{t}<10 \mathrm{~ns})$ within each of the three pores considered. In addition, the locations occupied or traversed by methane molecules generally show lower SFE because the presence of methane precludes many water molecules from occupying those regions as well. Methane trajectories are reported at various observation times together with the SFE map averaged over 2100 ps (top left), 2102.5 ps (top middle), and 2115 ps (top right) simulation fragments from an initial configuration for silica; over 2305 ps (middle left), 2315 ps (middle middle) and 2320 ps (middle right) for $\mathrm{MgO}$; and 17426 ps (bottom left), 17501 ps (bottom middle), 17526 ps (bottom right) for alumina. Visual inspection of the results in Figure 4 suggests that methane molecules travel larger distances when they enter low-density SFE regions, while they remain for longer times near high-density SFE locations, where perhaps they are trapped, waiting for a change in density fluctuation to open up possible diffusion pathways. Methane molecules generally avoid high-density SFE regions, resulting in vibratory movement when they are confined in relatively stable positions surrounded by high-density SFE. The resultant diffusion follows hopping events (some of which are shown as Supporting Information) from one favorable location to another. It appears that while the SFE distribution affects methane diffusion, the vice versa is also true, with the motion of methane molecules inside the pores saturated with water causing the evolution of SFE distributions over time.

\section{Transport of gas mixtures}

Because of the host-guest coupling between gas molecules and hydration water, it is likely that the transport behavior of various molecules through hydrated narrow pores will depend strongly on the 
properties of the interactions between the individual gas molecules and hydration water, possibly mediated via the constraints due to the solid substrate. To begin the exploration of these effects we conducted non-equilibrium simulations for the transport of gaseous mixtures through the three hydrated pores. In Figure 5 we report both the schematics for the simulated systems and the cumulative amount of gas collected in the permeate phase versus time for the hydrated silica (left), $\mathrm{MgO}$ (middle), and alumina (right) pores. In all cases, the methane : ethane : $\mathrm{H}_{2} \mathrm{~S}$ molar composition in the feed was of $8: 1.5: 0.5$. The results of gas fluxes are determined by the slope of the fitted straight lines (steady state). Combining the gas flux and the pressure drop (also shown in Figure 5) we obtain the permeability of methane, ethane, and $\mathrm{H}_{2} \mathrm{~S}$ using Eq. (2). The results show that $\mathrm{H}_{2} \mathrm{~S}$ permeates much more strongly the three hydrated pores than the other two components. The $\mathrm{H}_{2} \mathrm{~S} /$ methane and the $\mathrm{H}_{2} \mathrm{~S} /$ ethane selectivity, defined by the ratio of the permeabilities, we obtain: $\sim 31$ and $\sim 112$ for hydrated silica, $\sim 66$ and $\sim 56$ for hydrated $\mathrm{MgO}$, and $\sim 87$ and $\sim 59$ for hydrated alumina pores, respectively.
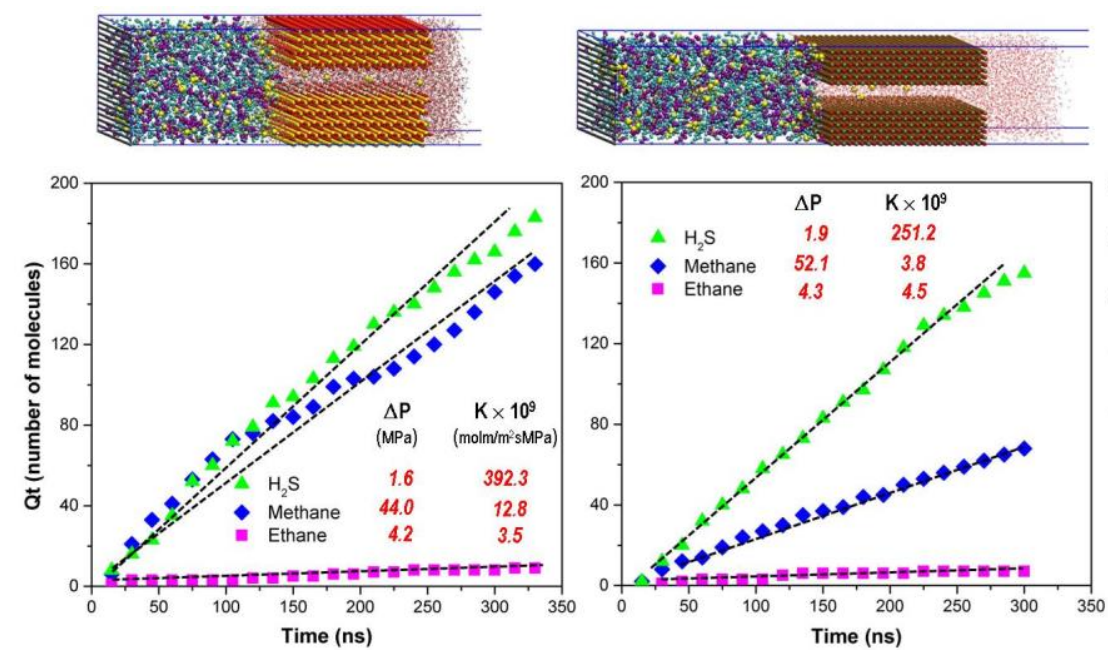
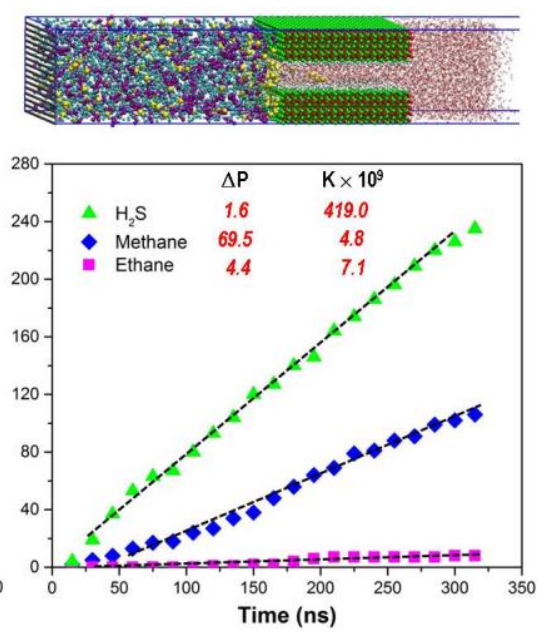

Figure 5. Top: Snapshots representing the simulated systems. Bottom: Cumulative number of gas molecules $\left(Q_{t}\right)$ in the permeate region as a function of time. Results are obtained for gas moving across the hydrated silica (left), hydrated $\mathrm{MgO}$ (middle), and hydrated alumina pores (right). The total number of gas molecules initially inserted in the three simulated systems was 3000, 3400, and 3800 for the silica, $\mathrm{MgO}$, and alumina systems, respectively. In all cases, the methane : ethane : $\mathrm{H}_{2} \mathrm{~S}$ molar composition was of $8: 1.5: 0.5$. The gas flux is determined by the slope of the fitted straight lines (black dashed lines). 
The simulation results suggest that all pores considered in this study are selective to $\mathrm{H}_{2} \mathrm{~S}$, with the hydrated alumina pores being much more selective than the others. The predicted $\mathrm{H}_{2} \mathrm{~S} /$ methane selectivity for the hydrated alumina pore, $\sim 87$, is higher than those reported for commercial Pebax membranes,${ }^{55} 40$ 70 , and for highly siliceous zeolites, ${ }^{4} \sim 44$. Polymeric membranes based on polyurethane-ether and polyurea-ether block copolymers are being proposed as promising membrane materials ${ }^{56}$ their $\mathrm{H}_{2} \mathrm{~S} /$ methane selectivity at 293-308 $\mathrm{K}(75-100)$ is comparable to that predicted for the hydrated pores considered here. ${ }^{55}$ At similar operational temperatures, the hydrated alumina pore presented here is predicted to have comparable $\mathrm{H}_{2} \mathrm{~S} /$ methane selectivity but much higher $\mathrm{H}_{2} \mathrm{~S}$ permeability $\left(\sim 42 \times 10^{-8}\right.$ $\mathrm{molm} / \mathrm{m}^{2} \mathrm{sMPa}$ for the hydrated alumina pore compared to $3.48-7.47 \times 10^{-8} \mathrm{molm} / \mathrm{m}^{2} \mathrm{sMPa}$ for polymeric membranes ${ }^{55}$ ), which suggests a very promising possibility of using the hydrated pores as perm-selective membranes for natural gas sweetening. In these pores hydration water would be essential.

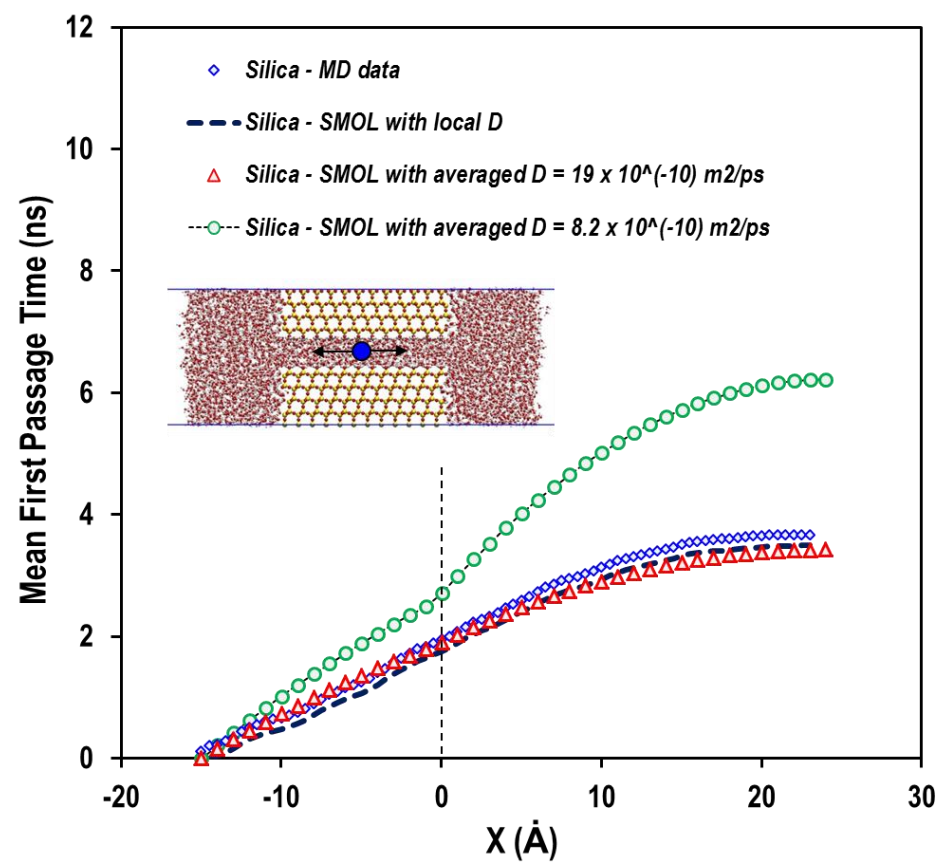

Figure 6. Mean first passage time (MFPT) profiles for methane to start at the center of the hydrated pores and reach the target $x_{t}$ outside the pores. (A) Results from explicit-water molecular dynamics (MD) simulations (blue diamonds) for methane moving across the hydrated silica pores are compared with those derived from the non- and inversion Smoluchowski (SMOL) approach (dashed dark blue). Also shown are the results from SMOL approach with the averaged self-diffusion obtained from SMOL approach (red triangles) and MSD calculations (green circles). The thickness of the pore is $\sim 5.1 \mathrm{~nm}$. The black dashed line represents the entrance of each pore. 


\section{$\underline{\text { Multi-scale considerations }}$}

The simulations discussed so far were conducted at the molecular scale, for pores of length much shorter than those found in any practical materials. To enable the investigation of practically important substrates, we require implementing multi-scale approaches that capture the important details of our simulations. For applications, the important parameter to be captured is the mean first passage time (MFPT). In Figure 6 we report MFPT results, which summarize averages collected during methane transport across the hydrated pores. For these calculations the tagged methane molecule was restricted to move onedimensionally along the $\mathrm{X}$ direction and it was placed at the center of the hydrated pore. The MFPT distribution was measured from explicit MD simulations during which the molecule was exiting from the pore (as shown in the inset); the time sequence was then transformed to obtain the MFPT for one methane molecule that reaches a position within the hydrated pore starting from outside of the pore. The MFPT profile from our MD simulations (blue diamonds) suggests that it takes methane 3.67 ns to reach the center of the hydrated silica pore starting from outside the pore $\left(x_{t} \sim-15 \dot{\mathrm{A}}\right)$. In our multi-scale approach the goal is to use local self-diffusion coefficients and free-energy profiles, obtained from explicit MD simulations, in a continuum model such as the Smoluchowski model of Eq. (7). By comparing the MFPT obtained from the continuum model to that calculated from explicit MD the robustness of the coarsegrained approach can be confirmed. Figure 6 presents the coarse-grained results obtained following two different methods:

(a) Predicted approach. We selected $D_{\mathrm{x}}=18 \times 10^{-10} \mathrm{~m}^{2} / \mathrm{s}$ for methane outside (as obtained using the position autocorrelation function method, see Ref. [15]) and $D_{x}=8.2 \times 10^{-10} \mathrm{~m}^{2} / \mathrm{s}$ for methane inside the pore (estimated from mean-square displacement (MSD) calculations for methane in a periodically infinite pore, see Table 2).

(b) Computationally intensive approach. Assuming that the dynamics of methane is strictly Markovian, the local self-diffusion $D(x)$ was calculated from the inversion of the Smoluchowski approach Eq. (8) (results are shown in Figure $\mathbf{S 4}$ of the Supporting Information) using PMF 
profiles and MD-derived MFPT as input (this $D(x)$ per construction reproduced the MD-derived MFPT). Then, we selected the calculated local self-diffusion of methane outside and inside the pore to correspond to the averaged data $D_{\text {out }}=25 \times 10^{-10} \mathrm{~m}^{2} / \mathrm{s}$ and $D_{\text {in }}=19 \times 10^{-10} \mathrm{~m}^{2} / \mathrm{s}$, as shown in Figure $\mathbf{S 4}$ of the Supporting Information, and used them to calculate the MFPT in Eq. (7).

We found that:

(a) The predicted approach yields a MFPT (green circles) that is $2.5 \mathrm{~ns}$ slower than the MFPT from MD data.

(b) Averaging the methane self-diffusion coefficients for inside and outside the pore based on the local self-diffusion profile $D(x)$ (red triangles) yields a very good agreement with the MD-derived MFPT. This indicates that the detailed structure of $D(x)$ is not crucial.

The results just described suggest that it is possible to describe methane transport through hydrated pores using a coarse-grained approach, but that the results depend strongly on the estimation of the selfdiffusion profile used as input. Unexpectedly the self-diffusion coefficients obtained from extensive MSD calculations such as those described in Table 2 yield data not in agreement with the diffusivity estimated from the inversion of the MD-derived MFPT. This could be explained by the observation reported by Zimmermann et al.: ${ }^{57}$ in a simulated system of reduced size the self-diffusion coefficients estimated by standard MSD methods can be greater than they would be in larger systems when diffusion is characterized by low energy barriers of the order of $0.6 \mathrm{kcal} / \mathrm{mol}$. In such conditions, molecules easily jump into the next cage and continue to carry out multi-jumps, which results in larger self-diffusivities in small simulation boxes than in larger ones. Because the diffusion mechanism discussed by Zimmermann et al. is similar to that observed for methane moving through hydrated pores, it is possible that the selfdiffusion coefficients reported in Table 2 for infinite pores are smaller than those we would obtain in smaller simulation boxes. Indeed, by conducting additional 100 independent simulations for one methane molecule confined in one hydrated silica pore of finite lateral size $(\sim 5.1 \mathrm{~nm}$, which corresponds to the size of the solid substrate used to calculate the MD-derived MFPT in Fig. 6), we obtain an averaged self- 
diffusivity of methane inside the pore of $\sim 18 \times 10^{-10} \mathrm{~m}^{2} / \mathrm{s}$ from MSD calculations. This value is consistent with the average of local self-diffusion profile $D(x)$ estimated using Eq. (8). The ability of the coarse-grained approach to reproduce the MD simulation results when an averaged value for the selfdiffusion coefficient is used as input confirms that the MFPT distribution can be accurately described by the Smoluchowski approach when reliable values for the self-diffusion coefficients are used as input, and when the pores are sufficiently long.

In Figure 7 we report the MFPT distribution for methane across hydrated silica (blue), MgO (green) and alumina (red) pores of length up to $\sim 40 \mathrm{~nm}$ as predicted by the coarse-grained approach just described using the self-diffusion data of Table 2 as input. The results show that methane diffuses through the hydrated silica pores much faster than through the other two pores. Because of computing power limitations, the verification of these predictions is left for future experimental studies. Such MFPT calculations could help predict the fate of methane through pore networks in subsurface formations, with possible implications for shale gas. For example, we estimate that to migrate across pores of length $1 \mathrm{~cm}$ methane molecules would require $1.25,2.5$, and 5 hours when the hydrated pores are carved out of silica, $\mathrm{MgO}$, and alumina, respectively. With accurate knowledge of the network of pores within fractured shale formations, similar calculations could help estimate the likelihood of methane to reach the water table. 


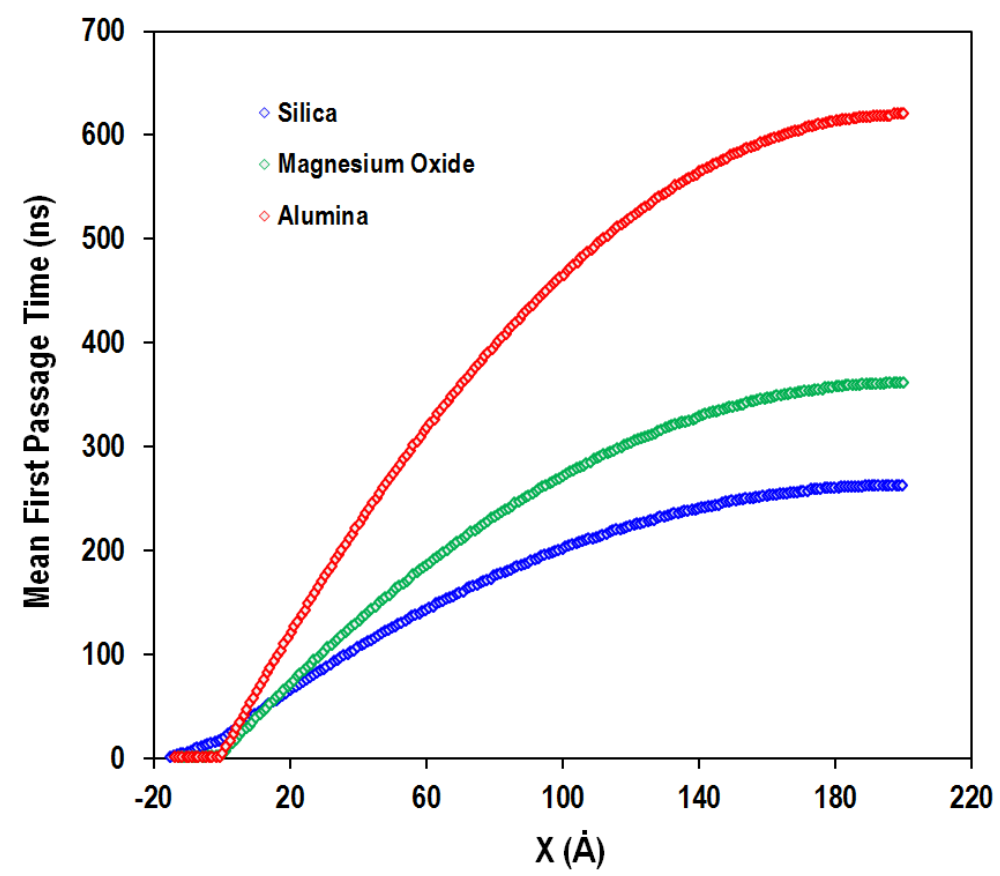

Figure 7. Estimated MFPT for methane to transport along hydrated pores carved out of silica (blue), $\mathrm{MgO}$ (green) and alumina (red) of length $40 \mathrm{~nm}$. The results are obtained by the Smoluchowski approach using the self-diffusion coefficients shown in

Table 2 as input.

\section{Conclusions}

Atomistic molecular dynamics simulations were employed to quantify the transport of methane, ethane, and $\mathrm{H}_{2} \mathrm{~S}$ through 1-nm wide slit-shaped pores filled with water at $300 \mathrm{~K}$. Advanced methods of sampling were implemented to identify the molecular features responsible for the observed impact of the hydration water structure on selective gas transport through the pores. It was found that the main impact on the transport of methane molecules inside the water-wet pores is the evolution of solvation free energy distribution within the pores. This property appears to be heterogeneous both in space and in time, despite the fact that the simulated systems have a very controlled spatially uniform solid structure. The hydration structure within narrow pores leads to significant selectivity to the hydrogen sulfide permeation as opposed to volatile hydrocarbon gases, with performance indicators comparable to, or even more promising than those reported for the current state-of-the-art polymeric membranes. This study suggests that a coarse-grained approach based on the Smoluchowski equation can be used to predict the mean first 
passage time for gas molecules through the hydrated pores, provided that a reliable value for the local self-diffusion profile is used for the calculations. The resultant model allows for estimations of gas transport through macroscopic pores requiring moderate computational resources. While experimental verification is required, the results revealed unexpected physical mechanisms responsible for gas molecules transport through hydrated systems that could be exploited for designing new membranes for natural gas sweetening and for assessing the likelihood of methane migrating from shale formations upon hydraulic fracturing operations. For the latter application, temperature effects on the phenomena described herein should be quantified thoroughly.

\section{Acknowledgements}

Generous allocations of computing time were provided by the University College London Research Computing Platforms Support (LEGION), the Oklahoma Supercomputing Center for Education and Research (OSCER) and the National Energy Research Scientific Computing Center (NERSC) at Lawrence Berkeley National Laboratory. NERSC is supported by the DOE Office of Science under Contract No. DE-AC02-05CH11231. Ms. Anh Phan wishes to thank financial support from the Sloan Foundation, via the Deep Carbon Observatory and by a Marie Curie Career Initiation Grant awarded to AS. RGW and JD thank the Deutsche Forschungsgemeinschaft (DFG) for financial support. DRC was supported by the Department of Energy, Office of Basic Energy Sciences, Geosciences program under grant DE-SC0006878. This research received funding from the European Union's Horizon 2020 research and innovation program under grant agreement No. 640979.

\section{Associated Content}

Supporting Information

Results for methane flux through hydrated pores, time correlation function for heterogeneity of solvation free energy, diffusion of methane in the hydrated pores, local self-diffusion profile, and transport properties of methane and gas mixtures through pores. 
This material is available free of charge via the Internet at http://pubs.acs.org.

\section{References}

(1) Katz, D. L. V. Handbook of Natural Gas Engineering; McGraw-Hill: New York, 1959.

(2) Kohl, A. L.; Nielsen, R. Gas Purification; Gulf Professional Publishing: Houston, TX, 1997.

(3) Robb, W. L. Thin Silicone Membranes-Their Permeation Properties and Some Applications. Annals of the New York Academy of Sciences 1968, 146, 119-137.

(4) Shah, M. S.; Tsapatsis, M.; Siepmann, J. I. Monte Carlo Simulations Probing the Adsorptive Separation of Hydrogen Sulfide/Methane Mixtures Using All-Silica Zeolites. Langmuir 2015, 31, 12268-12278.

(5) Amo, K. D.; Baker, R. W.; Lokhandwala, K. A.; Toy, L. G.; Membrane Technology and Research Inc., Menlo Park, Calif: US, 1995; Vol. 5,401,300.

(6) Vaughn, J. T.; Koros, W. J. Analysis of feed stream acid gas concentration effects on the transport properties and separation performance of polymeric membranes for natural gas sweetening: A comparison between a glassy and rubbery polymer. J Membrane Sci 2014, 465, 107-116.

(7) Jackson, R. B.; Vengosh, A.; Darrah, T. H.; Warner, N. R.; Down, A.; Poreda, R. J.; Osborn, S. G.; Zhao, K. G.; Karr, J. D. Increased stray gas abundance in a subset of drinking water wells near Marcellus shale gas extraction. P Natl Acad Sci USA 2013, 110, 11250-11255.

(8) King, G. E. In SPE Hydraulic Fracturing Technology Conference The Woodlands, Texas, U.S.A, 2012; Vol. SPE 152596, p 1-80.

(9) Bahrami, H.; Rezaee, R.; Clennell, B. Water blocking damage in hydraulically fractured tight sand gas reservoirs: An example from Perth Basin, Western Australia. J Petrol Sci Eng 2012, 88-89, 100-106.

(10) Bennion, D. B.; Thomas, F. B. Formation damage issues impacting the productivity of low permeability, low initial water saturation gas producing formations. J Energ Resour-Asme 2005, 127, 240-247.

(11) Phan, A.; Cole, D. R.; Striolo, A. Aqueous Methane in Slit-Shaped Silica Nanopores: High Solubility and Traces of Hydrates. J Phys Chem C 2014, 118, 4860-4868.

(12) Miachon, S.; Syakaev, V. V.; Rakhmatullin, A.; Pera-Titus, M.; Caldarelli, S.; Dalmon, J. A. Higher gas solubility in nanoliquids? Chemphyschem 2008, 9, 78-82.

(13) Luzar, A.; Bratko, D. Gas solubility in hydrophobic confinement. J Phys Chem B 2005, $109,22545-22552$.

(14) Phan, A.; Ho, T. A.; Cole, D. R.; Striolo, A. Molecular Structure and Dynamics in Thin Water Films at Metal Oxide Surfaces: Magnesium, Aluminum, and Silicon Oxide Surfaces. J Phys Chem $C$ 2012, 116, 15962-15973.

(15) Phan, A.; Cole, D. R.; Striolo, A. Factors Governing the Behavior of Aqueous Methane in Narrow Pores. Philosophical Transactions of the Royal Society A 2015, 374, 20150019.

(16) Ho, T. A.; Argyris, D.; Papavassiliou, D. V.; Striolo, A.; Lee, L. L.; Cole, D. R. Interfacial water on crystalline silica: a comparative molecular dynamics simulation study. Mol Simulat 2011, 37, 172-195.

(17) Argyris, D.; Tummala, N. R.; Striolo, A.; Cole, D. R. Molecular structure and dynamics in thin water films at the silica and graphite surfaces. J Phys Chem C 2008, 112, 13587-13599.

(18) Argyris, D.; Ho, T. A.; Cole, D. R.; Striolo, A. Molecular Dynamics Studies of Interfacial Water at the Alumina Surface. J Phys Chem C 2011, 115, 2038-2046. 
(19) Phan, A.; Cole, D. R.; Striolo, A. Preferential Adsorption from Liquid Water-Ethanol Mixtures in Alumina Pores. Langmuir 2014, 30, 8066-8077.

(20) Causa, M.; Dovesi, R.; Pisani, C.; Roetti, C. Abinitio Hartree-Fock Study of the Mgo(001) Surface. Surf Sci 1986, 175, 551-560.

(21) Scamehorn, C. A.; Hess, A. C.; Mccarthy, M. I. Correlation Corrected Periodic HartreeFock Study of the Interactions between Water and the (001) Magnesium-Oxide Surface. J Chem Phys 1993, 99, 2786-2795.

(22) Refson, K.; Wogelius, R. A.; Eraser, D. G.; Payne, M. C.; Lee, M. H.; Milman, V. Water Chemisorption and Reconstruction of the Mgo Surface. Phys Rev B 1995, 52, 10823-10826.

(23) Cygan, R. T.; Liang, J. J.; Kalinichev, A. G. Molecular models of hydroxide, oxyhydroxide, and clay phases and the development of a general force field. J Phys Chem B 2004, 108, 1255-1266.

(24) Martin, M. G.; Siepmann, J. I. Transferable potentials for phase equilibria. 1. Unitedatom description of n-alkanes. J Phys Chem B 1998, 102, 2569-2577.

(25) Berendsen, H. J. C.; Grigera, J. R.; Straatsma, T. P. The Missing Term in Effective Pair Potentials. J Phys Chem-Us 1987, 91, 6269-6271.

(26) Kamath, G.; Lubna, N.; Potoff, J. J. Effect of partial charge parametrization on the fluid phase behavior of hydrogen sulfide. J Chem Phys 2005, 123.

(27) Miyamoto, S.; Kollman, P. A. Settle - an Analytical Version of the Shake and Rattle Algorithm for Rigid Water Models. J Comput Chem 1992, 13, 952-962.

(28) Essmann, U.; Perera, L.; Berkowitz, M. L.; Darden, T.; Lee, H.; Pedersen, L. G. A Smooth Particle Mesh Ewald Method. J Chem Phys 1995, 103, 8577-8593.

(29) Allen, M. P.; Tildesley, D. J. Computer Simulation of Liquids; Oxford University Press: Oxford, UK, 2004.

(30) Cabrales-Navarro, F. A.; Gomez-Ballesteros, J. L.; Balbuena, P. B. Molecular dynamics simulations of metal-organic frameworks as membranes for gas mixtures separation. $J$ Membrane Sci 2013, 428, 241-250.

(31) Mizukami, K.; Takaba, H.; Kobayashi, Y.; Oumi, Y.; Belosludov, R. V.; Takami, S.; Kubo, M.; Miyamoto, A. Molecular dynamics calculations of $\mathrm{CO} 2 / \mathrm{N}-2$ mixture through the NaY type zeolite membrane. J Membrane Sci 2001, 188, 21-28.

(32) Yin, K. L.; Xu, D. J. Study on permeation of acetone/nitrogen mixed gas through Al2O3 microporous membranes: Molecular dynamics. Chem Eng Commun 2006, 193, 1678-1688.

(33) Golebiowska, M.; Roth, M.; Firlej, L.; Kuchta, B.; Wexler, C. The reversibility of the adsorption of methane-methyl mercaptan mixtures in nanoporous carbon. Carbon 2012, 50, 225-234.

(34) Park, I.; Knaebel, K. S. Adsorption Breakthrough Behavior - Unusual Effects and Possible Causes. Aiche J 1992, 38, 660-670.

(35) Hess, B.; Kutzner, C.; van der Spoel, D.; Lindahl, E. GROMACS 4: Algorithms for highly efficient, load-balanced, and scalable molecular simulation. J Chem Theory Comput 2008, 4, 435-447.

(36) Van der Spoel, D.; Lindahl, E.; Hess, B.; Groenhof, G.; Mark, A. E.; Berendsen, H. J. C. GROMACS: Fast, flexible, and free. J Comput Chem 2005, 26, 1701-1718.

(37) Hoover, W. G. Canonical Dynamics - Equilibrium Phase-Space Distributions. Phys Rev A $1985,31,1695-1697$.

(38) Nose, S. A Molecular-Dynamics Method for Simulations in the Canonical Ensemble. Mol Phys 1984, 52, 255-268.

(39) Hockney, R. W.; Goel, S. P.; Eastwood, J. W. Quiet High-Resolution Computer Models of a Plasma. J Comput Phys 1974, 14, 148-158.

(40) Rutherford, S. W.; Do, D. D. Review of time lag permeation technique as a method for characterisation of porous media and membranes. Adsorption 1997, 3, 283-312. 
(41) Daynes, H. A. The process of diffusion through a rubber membrane. $P R$ Soc Lond $a-$ Conta 1920, 97, 286-306.

(42) Barrer, R. M. Permeation, diffusion and solution of gases in organic polymers. T Faraday Soc 1939, 35, 0628-0643.

(43) Adams, R.; Carson, C.; Ward, J.; Tannenbaum, R.; Koros, W. Metal organic framework mixed matrix membranes for gas separations. Micropor Mesopor Mat 2010, 131, 13-20.

(44) Gubbins, K. E.; Liu, Y. C.; Moore, J. D.; Palmer, J. C. The role of molecular modeling in confined systems: impact and prospects. Phys Chem Chem Phys 2011, 13, 58-85.

(45) Ritos, K.; Mattia, D.; Calabro, F.; Reese, J. M. Flow enhancement in nanotubes of different materials and lengths. J Chem Phys 2014, 140.

(46) Torrie, G. M.; Valleau, J. P. Monte-Carlo Free-Energy Estimates Using Non-Boltzmann Sampling - Application to Subcritical Lennard-Jones Fluid. Chem Phys Lett 1974, 28, 578-581.

(47) Kumar, S.; Bouzida, D.; Swendsen, R. H.; Kollman, P. A.; Rosenberg, J. M. The Weighted Histogram Analysis Method for Free-Energy Calculations on Biomolecules .1. The Method. J Comput Chem 1992, 13, 1011-1021.

(48) Argyris, D.; Phan, A.; Striolo, A.; Ashby, P. D. Hydration Structure at the alpha-Al2O3 (0001) Surface: Insights from Experimental Atomic Force Spectroscopic Data and Atomistic Molecular Dynamics Simulations. J Phys Chem C 2013, 117, 10433-10444.

(49) Limmer, D. T.; Willard, A. P.; Madden, P.; Chandler, D. Hydration of metal surfaces can be dynamically heterogeneous and hydrophobic. P Natl Acad Sci USA 2013, 110, 4200-4205.

(50) Hummer, G.; Garde, S.; Garcia, A. E.; Pohorille, A.; Pratt, L. R. An information theory model of hydrophobic interactions. P Natl Acad Sci USA 1996, 93, 8951-8955.

(51) Hinczewski, M.; von Hansen, Y.; Dzubiella, J.; Netz, R. R. How the diffusivity profile reduces the arbitrariness of protein folding free energies. J Chem Phys 2010, 132.

(52) Sedlmeier, F.; von Hansen, Y.; Mengyu, L.; Horinek, D.; Netz, R. R. Water Dynamics at Interfaces and Solutes: Disentangling Free Energy and Diffusivity Contributions. J Stat Phys 2011, 145, 240-252.

(53) Marti, J. Analysis of the hydrogen bonding and vibrational spectra of supercritical model water by molecular dynamics simulations. J Chem Phys 1999, 110, 6876-6886.

(54) Witherspoon, P. A.; Saraf, D. N. Diffusion of Methane Ethane Propane and N-Butane in Water from 25 to 43 Degrees. J Phys Chem-Us 1965, 69, 3752-3755.

(55) Chatterjee, G.; Houde, A. A.; Stern, S. A. Poly(ether urethane) and poly(ether urethane urea) membranes with high H2S/CH4 selectivity. J Membrane Sci 1997, 135, 99-106.

(56) Simmons, J. W.; L'air Liquide-Societe Anonyme A'directoire Et Conseil De Surveillance Pour L'etude Et L'exploitation Des Procedures Georges Claude, Paris: US, 2005; Vol. US 6,843,829 B2.

(57) Zimmermann, N. E. R.; Zabel, T. J.; Keil, F. J. Transport into Nanosheets: Diffusion Equations Put to Test. J Phys Chem C 2013, 117, 7384-7390. 
TOC figure: Representative simulation snapshot for gas mixture transport through a hydrated pore, combined with in-plane solvation free energy distribution coupled with trajectories of representative guest molecules.

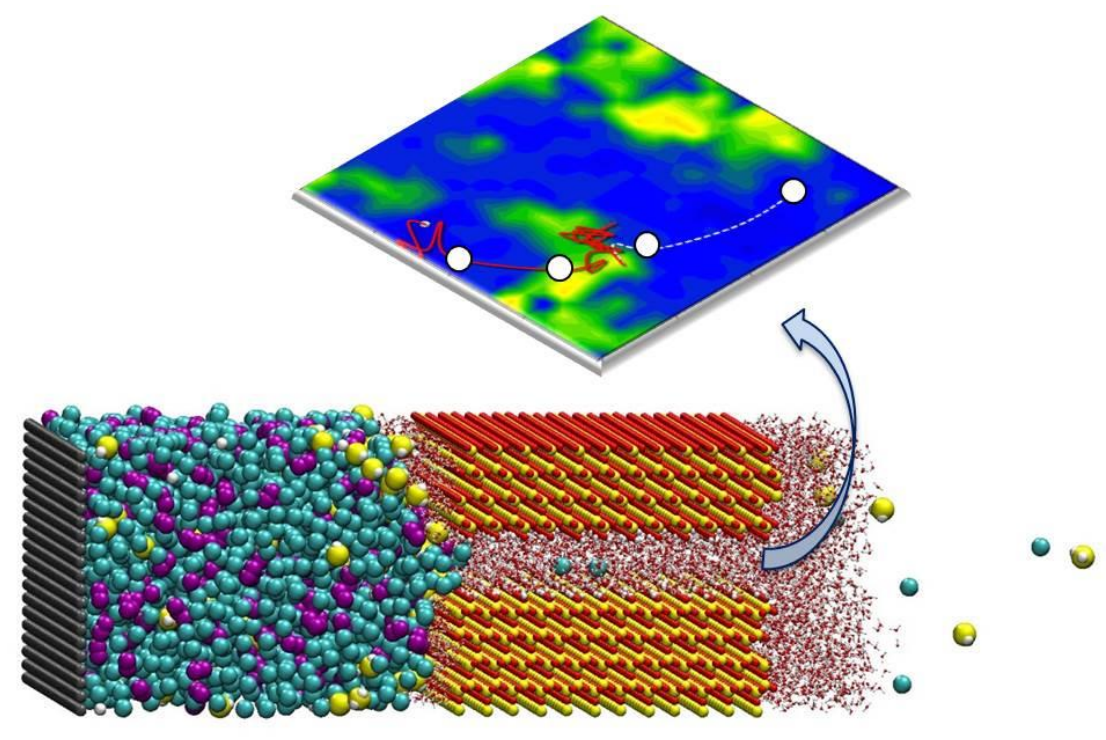

